\title{
Management of Intra-abdominal Infections due to Carbapenemase-Producing Organisms
}

\author{
Paola Di Carlo • Francesco Vitale • \\ Criostóir Ó'Súilleabháin • Alessandra Casuccio
}

(C) Springer Science+Business Media New York 2014

\begin{abstract}
The prevalence of bacterial resistance to carbapenem antibiotics continues to increase because of bacteria producing metallo- $\beta$-lactamases (MBL), called carbapenemaseproducing organisms (CPO). Enterobacteriaceae, which can be a common cause of intra-abdominal infections (IAIs), have become carbapenem-resistant Enterobacteriaceae (CRE). Updated international guidelines for the treatment of both IAIs and IAIs due to CRE have been published. Given the multifaceted nature of these infections, these recommendations have been jointly reviewed and endorsed by the Surgical Society and the Association of Medical Microbiology and Infectious Disease. The aims of this review are to summarize the general and new generation of multimodal procedure to manage IAIs due to CRE and review the data available on the combination of interventions to reduce CRE. Future research should focus on the development of novel and safe antimicrobial therapies and the quantification of the incremental effect of infection control programmes and new methods to rapidly detect pathogens before patients enter the surgical setting.
\end{abstract}

This article is part of the Topical Collection on Intra-abdominal Infections, Hepatitis, and Gastroenteritis

\author{
P. Di Carlo $\cdot$ F. Vitale $\cdot$ A. Casuccio $(\square)$ \\ Department of Sciences for Health Promotion and Mother-Child \\ Care "G. D'Alessandro", University of Palermo, Via del Vespro 127, \\ 90127 Palermo, Italy \\ e-mail: alessandra.casuccio@unipa.it \\ P. Di Carlo \\ e-mail: paola.dicarlo@unipa.it \\ F. Vitale \\ e-mail: francesco.vitale@unipa.it \\ C. Ó’Súilleabháin \\ Mercy University Hospital, Grenville Place, Cork, Ireland \\ e-mail: cbosullivan@muh.ie
}

Keywords Intra-abdominal infections .

Carbapenemase-producing organisms $\cdot$ Carbapenem-resistant Enterobacteriaceae $\cdot$ Management of intra-abdominal infections

\section{Introduction}

Abdominal surgery carries significant morbidity and mortality, which is in turn associated with an enormous use of healthcare resources. Intra-abdominal infections are particularly significant as they occur relatively frequently and have potentially serious consequences; they can slow down patient recovery, prolong hospital stay, and require greater antibiotic use. Intraabdominal infections (IAIs) comprise a wide variety of specific infections, ranging from uncomplicated appendicitis to cholecystitis and faecal peritonitis, and are therefore classified as either uncomplicated or complicated such as intraabdominal abscess or peritonitis. In the majority of uncomplicated IAIs, definitive management is surgical and antibiotics are not warranted beyond prophylaxis of the incision against SSI. However, in certain instances, the pathogens extend beyond the source organ and into the peritoneal cavity through a perforated viscus, thereby stimulating the systemic inflammatory response to a greater degree. The extent of such infection depends upon the combination of local host defences and the virulence of microorganisms [1].

Pyogenic liver abscesses usually develop following peritonitis due to an intra-abdominal abscess that subsequently spreads to the liver via the portal circulation or via direct spread from a biliary infection. They may also result from arterial haematogenous seeding in the setting of a systemic infection.

In such cases, sepsis is a serious complication and mortality is approximately $25-35 \%[1-3,4 \cdot]$ but may 
exceed $80 \%$ if the infection is caused by multidrugresistant (MDR) microorganisms [5••, 6•, 7-9].

The management of patients with MDR infections often requires the involvement of multiple medical disciplines. Inadequate surgical source control of an intra-abdominal infection with lack of control of the underlying pathological process by resection, closure or drainage, or drainage of existing purulent collections are causes of therapeutic failure. Selection of an ineffective antimicrobial agent is another cause of therapeutic failure $[7,8,10]$.

Resistance to carbapenems has been described as naturally occurring in several Gram-positive and Gram-negative bacterial species producing metallo- $\beta$-lactamases (MBL). This phenotypic grouping of enzymes confers resistance to all $\beta$ lactams except monobactams and is not susceptible to the clinically available $\beta$-lactamase inhibitors.

Several types of these enzymes have been detected, namely IMP, VIM, SPM, GIM, and SIM, mostly in Pseudomonas aeruginosa and other Gram-negative nonfermenters [11••, 12]. Moreover, the two VIM-producing Enterobacteriaceae identified in the USA were the same strains isolated from patients previously hospitalized in other southern European countries such as Greece and Italy [13-15].

\section{Carbapenem-Resistant Enterobacteriaceae}

Enterobacteriaceae are the predominant microbial species of bacterial flora in the human gut and also a frequent cause of IAIs. Therefore, the increased resistance among the Enterobacteriaceae to antimicrobial agents has resulted in the increasing frequency of IAIs caused by MDR strains [10, 16]. As a result, restricting more and more the treatment options and the more frequent use of broad-spectrum carbapenem agents as a last chance for effective therapy have inevitably resulted in the emergence and spread of carbapenem-resistant Enterobacteriaceae (CRE), and this has become a serious public health problem over the past decade.

Enterobacteriaceae can be resistant to carbapenems by mechanisms other than a carbapenemase, the most common of which is expression of an extended-spectrum cephalosporinase, such as an AmpC-type enzyme or an extended-spectrum beta-lactamase (ESBL), combined with porin loss. Of major concern is the coexistence of multiple ESBL and carbapenemase genes as well as that of other antibiotic resistance determinants on mobile elements that, along with the genetic plasticity of the Enterobacteriaceae, has led to rampant intra- and interspecies transfer of these elements and emergence of organisms with resistance to virtually all antibiotics $[17,18]$.

Among CRE, virulent clones of Klebsiella pneumoniae in particular have been described as an emerging causative agent of complicated intra-abdominal infections such as liver or pancreatic abscesses $[19,20]$. Carbapenem resistance in $K$. pneumoniae can be mediated by the production of carbapenemases belonging to Ambler class A, B, or D. The most common carbapenemase worldwide is $K$. pneumoniae carbapenemase (KPC), an Ambler molecular class A enzyme that hydrolyses a broad variety of $\beta$-lactams. In addition, KPC-producing isolates demonstrate resistance to many agents commonly used to treat Gram-negative infections, including quinolones, and aminoglycosides. The globally distributed sequence type (ST) of $K$. pneumoniae associated with KPC enzyme production is ST258. However, despite the internationally prominent role of ST258, other $K$. pneumoniae STs reported as carrying KPC include STs $14,21,37,45,101$, 228, 234, 257, and 259 in the USA and STs 277, 327, 340 , and 376 in Israel. The potential for the spread of plasmid-encoded KPC carbapenemases to different strains, including those locally more prevalent, is a serious cause of concern $[9,21-23]$.

\section{Management of Intra-abdominal Infections due to CRE}

\section{General Recommendations}

Many strategies have been developed to manage intraabdominal infections due to CRE [24, 25]. Given the multifaceted nature of these infections, recommendations have been jointly developed and endorsed by the Surgical Society and the Association of Medical Microbiology and Infectious Disease. However, no guideline can take into account all the variations in ecology, patterns of antimicrobial resistance, patient characteristics, and organizational models of health care in different countries.

Antimicrobial therapy should be initiated once a patient receives a diagnosis of an intra-abdominal infection or once such an infection is considered likely. For patients with septic shock, antibiotics should be administered as soon as possible.

Antimicrobial therapy strictly depends upon monitoring in vitro antimicrobial susceptibility using the clinical breakpoints defined by the CLSI guidelines for the detection of isolates producing carbapenemases [26] and the European Committee on Antimicrobial Susceptibility Testing (EUCAST) [27].

Until now, the detection of carbapenemase production has been complicated because some carbapenemase-producing isolates demonstrate elevated but susceptible carbapenem MICs. In fact, variability in the performance of phenotypic tests for detection of MBLs and difficulty with their interpretation have been described previously [28], and our reports demonstrate the need to include multiple methods to ensure reliable detection of MBLs. The Etest is a feasible option, but MBL could demonstrate only a subtle deformation of the 
ellipse in the K. pneumoniae parent and Escherichia coli transformant strains [29].

\section{Specific Antimicrobial Treatment}

Colistin and tigecycline have recently been granted a major role in the treatment of the most problematic MDR Gramnegative pathogens (ESBL- and carbapenemase-producing strains). The progressive increase of these pathogens has called for a re-evaluation of old antimicrobials such as fosfomycin, doxycycline, and chloramphenicol and new drugs such as temocillin $[30,31]$. Investigation on their activity against CRE showed a good activity against individual isolates [32-34] but rarely a bactericidal effect against carbapenemase-producing K. pneumoniae for which a double or triple combination of antibiotics is suggested [35].

Previous studies have reported them being used as a monotherapy for patients with serious polymicrobial infections, including MDR microorganisms [20, 30, 36]. Both antibiotics show favourable penetration into various tissues, such as the sites of infection and soft tissues of the skin, as well as intraabdominal infections (official indications). A review of current literature [21] concludes that clinical success rates are low when polymyxins (e.g. colistin) are used as monotherapy but higher when they are used in combination with tigecycline or gentamicin [21]. Accordingly, combined tigecycline and colistin has shown good in vitro synergy against carbapenemresistant Acinetobacter baumannii with a high-level imipenem resistance [37].

In our study [9], we report on the use of high-dose tigecycline in patients with intra-abdominal abscess due to carbapenemase-producing K. pneumoniae, especially in those with a less favourable clinical course and with KPC isolates showing MICs of tigecycline near the upper limit of the EUCAST susceptibility range (http://www.eucast.org). [27]

In addition, the role of combination therapy, including carbapenem-containing regimens investigated by in vitro time-kill experiments, remains to be defined [38]. The main therapeutic strategies cited in the literature for CRE infection clinical management are reported in Table 1.

\section{Current Practice Procedures for the Management} of Intra-abdominal Infections due to CRE

Selective decontamination of the digestive tract (SDD) reduces the number of Gram-negative and yeast infections in intensive care patients and reduces hospital mortality $[52,53]$. It does so by eradicating Gram-negative bacteria and yeasts from the intestinal tract, while preserving Gram-positive and anaerobic bacteria. Treatment consists of the local application of tobramycin, polymyxin, and amphotericin in the oropharynx and gastrointestinal tract. If the gut barrier is intact, the antibiotics exert their effect in the digestive tract and are not absorbed. However, conditions such as sepsis, shock, and major surgery can lead to gut barrier failure [54] and may result in the antibiotics leaking from the gut into the blood.

Experience with known antibiotic classes provides established benchmarks for new class members. The nextgeneration aminoglycoside plazomicin retains activity against all isolates of $K$. pneumoniae, E. coli, and Enterobacter spp. tested, including those with ESBL, KPC, and VIM-MBL resistance mechanisms, and may represent a promising alternative for the treatment of MDR pathogens. Pharmacokinetic and pharmacodynamic studies in animal models and in healthy volunteers have been started, and a pivotal phase 3 superiority trial of plazomicin for the treatment of serious CRE infections will be initiated in the first quarter of $2014[55,56]$.

New inhibitors of topoisomerases, cell wall synthesis, and protein synthesis are currently being developed. At this moment, in vitro and animal model studies have shown that they are well tolerated in animals and efficacious in Gram-negative infection models $[57,58]$.

Finally, the monoclonal antibody $(\mathrm{mAb})$ revolution offers many new options for the treatment of infectious diseases, though it is the subject of ongoing debate for various reasons, including the high cost of immunoglobulin preparations and relatively small markets. Clinical trials using $\mathrm{mAb}$ therapies are currently limited to treating Gram-positive bacteria and yeast infections [59, 60]. In this framework, nanotechnologybased solutions and, in particular, silver nanoparticles (AgNPs) have recently emerged as promising new antibacterial agents in the market [61•].

\section{Key Recommendations for Infection Control and Prevention}

CRE infections mostly occur in intensive care units and chronic care facilities. Transmission of carbapenem-resistant genes among bacterial species existing in the same environment contributes to the spread of resistance. Infection control and antibiotic stewardship remain our main lines of defence to limit propagation $[62 \bullet \bullet]$.

When CRE infections occur, they must be reported without delay and an investigation to determine the source initiated by a team of epidemiologists. Moreover, infection control staff should perform active surveillance screening, collecting rectal swabs from all the patients in units where microorganisms have been identified. The health-care personnel should also be instructed to screen and isolate patients with multidrugresistant organisms, as recommended by the Centers for Disease Control and Prevention (Atlanta).

Infection control measures should include strict contact precautions and more intensive cleaning practices. Standard infection control practices involve basic hand hygiene before and after unit entry with appropriate antimicrobial soaps; an aseptic technique when performing invasive procedures; the use of a gown, gloves, and mask for sterile procedures; routine 
Table 1 Antibiotic treatment indications for clinical management of CRE infection

\begin{tabular}{l} 
Monotherapy $\quad$ Combination therapy \\
\hline Colistin \\
3 MU every $8 \mathrm{~h}$, \\
loading dose of $6-9 \mathrm{MU}$, \\
followed by maintenance \\
doses of $4.5-6$ MU every \\
$12 \mathrm{~h}$-always adjusting to \\
renal function
\end{tabular}

Tigecycline,

$100 \mathrm{mg}$ IV loading, then

$50 \mathrm{mg}$ IV q12 h,

$200 \mathrm{mg}$ IV loading, then

$100 \mathrm{mg}$ IV q12 h

Fosfomycin

daily dose ranges from 12 to

$16 \mathrm{~g}$ on average,

administered in 2-4 infusions

a dosing interval of 6-8 h

should be preferred over $12 \mathrm{~h}$

\footnotetext{
Aminoglycosides

Amikacin 1,500-2,000 mg/

day every $24 \mathrm{~h}$

Gentamicin $7 \mathrm{mg} / \mathrm{kg}$ /day every $24 \mathrm{~h}$

Tetracycline

$100 \mathrm{mg} \mathrm{q} 12 \mathrm{~h}$ (maximum dose $300 \mathrm{mg}$ )

Chloramphenicol

$50 \mathrm{mg} / \mathrm{kg} /$ day in 4 divided

doses increased to

$100 \mathrm{mg} / \mathrm{kg} /$ day for severe infections
}

Colistin+tigecycline

Colistin+rifampicin

Colistin+carbapenem

Colistin + aminoglycosides

Colistin + fosfomycin

Colistin+rifampicin+ meropemen

Reference

Poulakou G et al., [37]

Daikos GL et al. [39]

Kontopidou F et al. [40]

Hirsch EB et al. [21], Sheng et al. [38]

$$
\begin{aligned}
& \text { Durante-Mangoni E } \\
& \text { et al. [41] } \\
& \text { Gaibani P et al. [42] }
\end{aligned}
$$

Peck KR et al. [32]

Akova M et al. [43]

Hirsch EB et al. [21]

Corvec S et al. [44]

Tängdén T et al. [35]

Bodmann KF et al. [4
Di Carlo P et al. [9]

In Europe, tromethamine derivative and fosfomycin calcium for oral use, as well as fosfomycin disodium for intravenous use, are available and marketed under different brand names.

\author{
[46] \\ Falagas ME et al. [47]
}

Flag

Colistin remains a valuable antimicrobial agent for treating these infections.
Tigecycline plus gentamicin or colistin was effective for treating 24 of 26 (92\%) Klebsiella pneumoniae carbapenemase-producing $K$. pneumoniae infectious episodes in 22 polytrauma intensive care unit patients without comorbidities.

A. baumannii infection mortality is not reduced by addition of rifampicin to colistin.

Combinations of colistin plus rifampicin, and less frequently tigecycline, exhibited synergistic activity in vitro against colistin-resistant KPC-Kp strains.

In cases where the MICs for carbapenems are not available or are higher than $8 \mathrm{mg} / \mathrm{l}$, this class of drugs should not be used as part of a combination regimen to avoid further selection of resistance.

Treatment with aminoglycosides polymyxin combinations have higher success rates compared to polymyxin monotherapy.

The combination of fosfomycin plus colistin showed the highest cure rate against CTX-M15-producing strain of Escherichia coli.

In multivariate analysis, combination therapy with tigecycline, colistin, and meropenem was independently associated with survival.

Trials with higher dosing schedules are eagerly awaited; favourable clinical success rates in a population of patients seriously ill with complicated intra-abdominal infections or complicated skin and skin tissue infections.

Fosfomycin should not be used as monotherapy to treat CRE infection for potential emergence of resistance during therapy.

Levy Hara G et al. [48] Typically, nephrotoxicity related to aminoglycoside use is reversible. Aminoglycosides should not be used as monotherapy to treat CRE infection especially as monotherapy against carbapenemase-producing $K$. pneumoniae.

Livermore DM et al. Carbapenem-resistant Enterobacteriaceae and, recently, [49]

Merino $\mathrm{M}$ et al. [50] outbreak of carbapenem-resistant Acinetobacter baumannii isolates showed susceptibility to minocycline.

Livermore DM et al. [49] Cuzon G et al. [51]
Carbapenem-resistant Enterobacteriaceae and, recently, outbreak of $E$. coli producing novel NDM carbapenemase showed susceptibility to minocycline. environmental cleaning and disinfection; and antibiotic stewardship. Flags indicating the presence of a multidrug-resistant organism should be placed in the electronic medical record and chart. 
Enhanced infection control practices include active surveillance (non-clinical) cultures of patients, staff, and the care environment, performed once or repeated at regular intervals, to detect asymptomatic carriage; geographic (spatial) cohorting of patients; geographic cohorting of staff; contact precautions (wearing a gown and gloves when entering the patient care area); dedicated patient equipment (i.e. glucometers and stethoscopes); un-identified unit observations of infection control practices; staff education; and regular consultation with infectious disease specialists. Additionally, in some centres, the use of periodic screening surveillance cultures has been recommended to better detect potentially unrecognized sources or reservoirs of resistant pathogens in the surgical setting.

The clinician's best strategy to manage nosocomial infections caused by CRE is to prevent infection or colonization of these organisms. A multifaceted approach to the treatment and management of these organisms when they are identified is needed, including strict adherence to enhanced infection control practices; active surveillance cultures of patients, staff, and the environment; and rapid switch to appropriate antimicrobial therapy upon receipt of the offending pathogen's antimicrobial susceptibility report, in order to better target therapy.

In Fig. 1 are shown the "Routine Practices and Additional Precautions in All Health Care Settings" reported by the Ontario Agency for Health Protection and Promotion, Provincial Infectious Diseases Advisory Committee [63].

Future research should focus on the development of novel and safe antimicrobial therapies which are active against CRE infections, evidence-based infection control practices, and new methods to rapidly detect pathogens before patients enter the surgical setting.

\section{Expert Opinion}

Recent epidemiological studies have shown the following:

- Owing to the limitation in efficacy and potential for toxicity of the alternative agents, many experts recommend using combination therapy instead of monotherapy in CRKP-infected patients. Nevertheless, in the absence of welldesigned comparative studies, the best combination to manage CRE infection, the continued role for carbapenems in combination therapy, and when combination therapy should be started, remain open questions.

- A lower-than-expected rate of health-care-associated infections based on estimates in other countries, due to a number of factors: diagnostic accuracy problems, differences in hospital patient case-mix, unsatisfactory surveillance system sensitivity, and incomplete and untimely reports.

- A very high frequency of infections in surgical patients especially in cancer patients; nevertheless, studies on infection control measures have shown that their incidence declines when control programmes are implemented.

- Preoperative markers of infection complications are important in high-risk populations such as older patients, because of frailty, disability, and co-morbidity.

- Decontamination to eradicate MDR-GNB should only be used in research settings and with detailed and real-time microbiological monitoring.

There are five ways to control multiresistant bacteria in your hospital setting:

- Prevent understaffing of nurses.

- Clean the skin.

- Screen and isolate.
Fig. 1 "Routine Practices and Additional Precautions in All Health Care Settings" (reported by the Ontario Agency for Health Protection and Promotion, Provincial Infectious Diseases Advisory Committee, 2013)

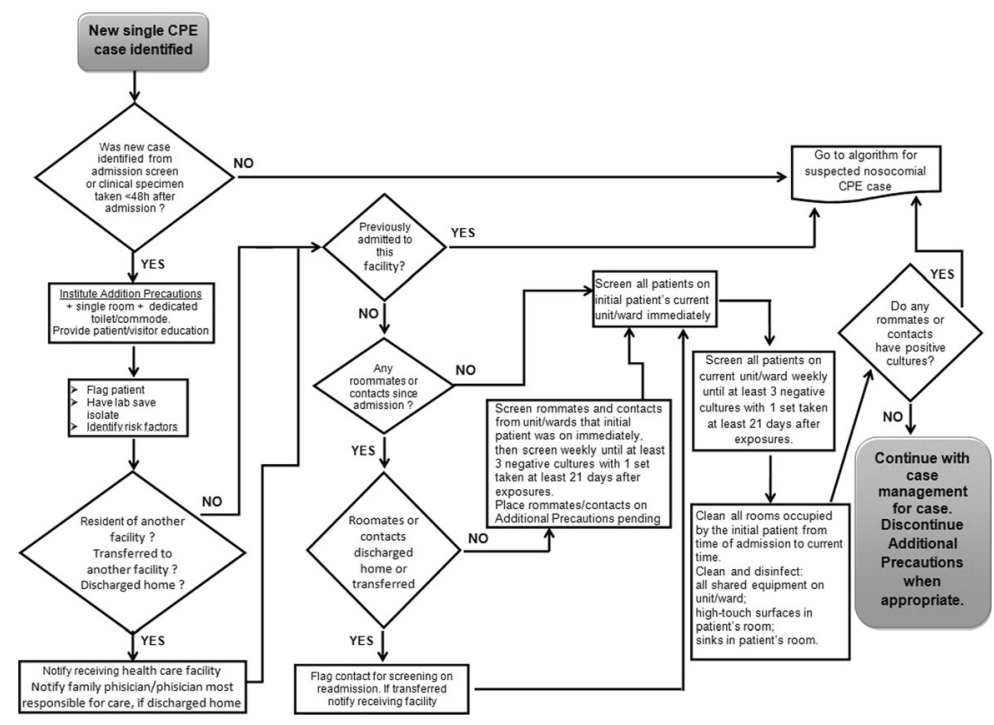


- Clean the environment.

- Limit unnecessary antibiotic use.

\section{Conclusions}

Intra-abdominal infections are particularly significant as they occur relatively frequently and have potentially serious consequences; they can slow down patient recovery, prolong hospital stay, and entail greater antibiotic use.

The management of patients with these infections often demands the involvement of multiple medical disciplines. Complicated infections are those that require both surgical or radiological drainage procedures and antimicrobial therapy. Inadequate surgical source control of an intra-abdominal infection with lack of control of the underlying pathological process by resection, closure or drainage, or drainage of existing purulent collections, are causes of therapeutic failure. Selection of an ineffective antimicrobial agent is another cause of therapeutic failure.

Our ability to prevent significant morbidity and mortality from intra-abdominal infections due to CRE remains a challenge.

Although antimicrobial prophylaxis has been shown to be effective in selected patient populations, there is still some debate on the application of risk prediction tools and other preventive strategies. There is a continued need for surveillance of CRE in order to develop and evaluate prevention strategies and to monitor changes in incidence and resistance.

\section{Compliance with Ethics Guidelines}

Conflict of Interest Paola Di Carlo, Francesco Vitale, Criostóir Ó'Súilleabháin, and Alessandra Casuccio declare that they have no conflicts of interest.

Human and Animal Rights and Informed Consent This article does not contain any studies with human or animal subjects performed by any of the authors.

\section{References}

Papers of particular interest, published recently, have been highlighted as:

- Of importance

-. Of major importance

1. O'Suilleabhain CB, Kim S, Rodrick MR, Mannick JA, Lederer JA. Injury induces alterations in T-cell NFkappaB and AP-1 activation. Shock. 2001;15:432-7.

2. Pieracci FM, Barie PS. Management of severe sepsis of abdominal origin. Scand J Surg. 2007;96:184-96.
3. Niven D, Laupland K, Tabah A, Vesin A, Rello J, Koulenti D, et al. Diagnosis and management of temperature abnormality in ICUs: a EUROBACT investigators survey. Crit Care. 2013;17:R289.

4. Moore LJ, Moore FA. Epidemiology of sepsis in surgical patients. Surg Clin North Am. 2012;92:1425-43. The article provides definitions and guidelines for the screening and management of sepsis and septic shock.

5.• Chen YH, Hsueh PR. Changing bacteriology of abdominal and surgical sepsis. Curr Opin Infect Dis. 2012;25:590-5. This review focuses on recent changes in epidemiological aspects of bacteriainduced intra-abdominal infections (IAIs).

6. Alexiou VG, Michalopoulos A, Makris GC, Peppas G, Samonis G, Falagas ME. Multi-drug-resistant gram-negative bacterial infection in surgical patients hospitalized in the ICU: a cohort study. Eur J Clin Microbiol Infect Dis. 2012;7-566. This study depicts certain, potentially modifiable, risk factors for postoperative infections in patients hospitalized in the ICU.

7. Zarkotou O, Pournaras S, Tselioti P, Dragoumanos V, Pitiriga V, Ranellou K, et al. Predictors of mortality in patients with bloodstream infections caused by KPC-producing Klebsiella pneumoniae and impact of appropriate antimicrobial treatment. Clin Microbiol Infect. 2011;17:1798-803.

8. Lee SS, Chen YS, Tsai HC, Wann SR, Lin HH, Huang CK, et al. Predictors of septic metastatic infection and mortality among patients with Klebsiella pneumoniae liver abscess. Clin Infect Dis. 2008;47:642-50.

9. Di Carlo P, Gulotta G, Casuccio A, Pantuso G, Raineri M, Farulla CA, et al. KPC - 3 Klebsiella pneumoniae ST258 clone infection in postoperative abdominal surgery patients in an intensive care setting: analysis of a case series of 30 patients. BMC Anesthesiol. 2013;3(1):13.

10. Solomkin JS, Mazuski J. Intra-abdominal sepsis: newer interventional and antimicrobial therapies. Infect Dis Clin North Am. 2009;23:593-608.

11.• Gupta N, Limbago BM, Patel JB, Kallen AJ. Carbapenem-resistant Enterobacteriaceae: epidemiology and prevention. Clin Infect Dis. 2011;53:60-7. This review describes the current epidemiology of CRE in the United States and highlights important prevention strategy.

12. Woodford N, Turton JF, Livermore DM. Multiresistant Gramnegative bacteria: the role of high-risk clones in the dissemination of antibiotic resistance. FEMS Microbiol Rev. 2011;35:736-55.

13. Lolans K, Queenan AM, Bush K, Sahud A, Quinn JP. First nosocomial outbreak of Pseudomonas aeruginosa producing an integron-borne metallo-beta-lactamase (VIM-2) in the United States. Antimicrob Agents Chemother. 2005;49:3538-40.

14. Vatopoulos A. High rates of metallo-beta-lactamase-producing Klebsiella pneumoniae in Greece - a review of the current evidence. Euro Surveill. 2008;13(4).

15. Mazzariol A, Mammina C, Koncan R, Di Gaetano V, Di Carlo P, Cipolla D, et al. A novel VIM-type metallo-beta-lactamase (VIM14) in a Pseudomonas aeruginosa clinical isolate from a neonatal intensive care unit. Clin Microbiol Infect. 2011;17:722-4.

16. Farr BM, Salgado CD, Karchmer TB, Sherertz RJ. Can antibioticresistant nosocomial infections be controlled? Lancet Infect Dis. 2001;1:38-45.

17. Bradford PA, Urban C, Mariano N, Projan SJ, Rahal JJ, Bush K. Imipenem resistance in Klebsiella pneumoniae is associated with the combination of ACT-1, a plasmid-mediated AmpC betalactamase, and the foss of an outer membrane protein. Antimicrob Agents Chemother. 1997;41:563-9.

18. Gazin M, Paasch F, Goossens H, Malhotra-Kumar S, MOSAR WP2 and SATURN WP1 Study Teams. Current trends in culturebased and molecular detection of extended-spectrum- $\beta$-lactamaseharboring and carbapenem-resistant Enterobacteriaceae. J Clin Microbiol. 2012;50:1140-6. 
19. Harada S, Tateda K, Mitsui H, Hattori Y, Okubo M, Kimura S, et al. Familial spread of a virulent clone of Klebsiella pneumoniae causing primary liver abscess. J Clin Microbiol. 2011;49:2354-6.

20. Di Carlo P, Pantuso G, Cusimano A, D'Arpa F, Giammanco A, Gulotta $\mathrm{G}$, et al. Two cases of monomicrobial intraabdominal abscesses due to KPC - 3 Klebsiella pneumoniae ST258 clone. BMC Gastroenterol. 2011;11:103.

21. Hirsch EB, Tam VH. Detection and treatment options for Klebsiella pneumoniae carbapenemases (KPCs): an emerging cause of multidrug-resistant infection. J Antimicrob Chemother. 2010;65: 1119-25.

22. Brisse S, Fevre C, Passet V, Issenhuth-Jeanjean S, Tournebize R, Diancourt L, et al. Virulent clones of Klebsiella pneumoniae: identification and evolutionary scenario based on genomic and phenotypic characterization. PLoS One. 2009;4(3):e4982.

23. Kanhutu KN, Post JJ, Clezy KR, Foo HY. Community-acquired Klebsiella pneumoniae liver abscesses an "emerging disease" in Australia. Med J Aust. 2011;194:374-7.

24. Centers for Disease Control and Prevention (CDC). Guidance for control of infections with carbapenem-resistant or carbapenemaseproducing Enterobacteriaceae in acute care facilities. MMWR Morb Mortal Wkly Rep. 2009;58(10):256-60.

25. Gastmeier P, Schwab F, Sohr D, Behnke M, Geffers C. Reproducibility of the surveillance effect to decrease nosocomial infection rates. Infect Control Hosp Epidemiol. 2009;30:993-9.

26. Clinical and Laboratory Standards Institute. Performance standards for antimicrobial susceptibility testing; 21 st informational supplement. M100-S21. Wayne: Clinical and Laboratory Standards Institute; 2011

27. European Committee on Antimicrobial Susceptibility Testing: clinical breakpoint Table 3.1. EUCAST. 2013 http://www.eucast.org/ fileadmin/src/media/PDFs/EUCAST_files/Disk test documents/ EUCAST breakpoints_v1.3 pdf.pdf

28. Mochon AB, Garner OB, Hindler JA, Krogstad P, Ward KW, Lewinski MA, et al. New Delhi metallo- $\beta$-lactamase (NDM-1)producing Klebsiella pneumoniae: case report and laboratory detection strategies. J Clin Microbiol. 2011;49:1667-70.

29. Limbago BM, Rasheed JK, Anderson KF, Zhu W, Kitchel B, Watz $\mathrm{N}$, et al. IMP-producing carbapenem-resistant Klebsiella pneumoniae in the United States. J Clin Microbiol. 2011;49: 4239-45.

30. Garau J. Other antimicrobials of interest in the era of extendedspectrum beta-lactamases: fosfomycin, nitrofurantoin and tigecycline. Clin Microbiol Infect. 2008;14 Suppl 1:198-202.

31. van Duin D, Kaye KS, Neuner EA, Bonomo RA. Carbapenemresistant Enterobacteriaceae: a review of treatment and outcomes. Diagn Microbiol Infect Dis. 2013;75(2):115-20.

32. Peck KR, Kim MJ, Choi JY, Kim HS, Kang CI, Cho YK, et al. In vitro time-kill studies of antimicrobial agents against blood isolates of imipenem-resistant Acinetobacter baumannii, including colistinor tigecycline-resistant isolates. J Med Microbiol. 2012;61(Pt 3): 353-60.

33. Porres-Osante N, Azcona-Gutiérrez JM, Rojo-Bezares B, Undabeitia E, Torres C, Sáenz Y. Emergence of a multiresistant KPC-3 and VIM-1 carbapenemase-producing Escherichia coli strain in Spain. J Antimicrob Chemother. 2014

34. Hasan B, Perveen K, Olsen B, Zahra R. Emergence of carbapenemresistant Acinetobacter baumannii in hospitals in Pakistan. J Med Microbiol. 2014;63(Pt 1):50-5.

35. Tängdén T, Hickman RA, Forsberg P, Lagerbäck P, Giske CG, Cars O. Evaluation of double- and triple-antibiotic combinations for VIM- and NDM-producing Klebsiella pneumoniae by in vitro time-kill experiments. Antimicrob Agents Chemother. 2014;58(3): 1757-62.

36. Kallel H, Bahloul M, Hergafi L, Akrout M, Ketata W, Chelly H, et al. Colistin as a salvage therapy for nosocomial infections caused by multidrug-resistant bacteria in the ICU. Int J Antimicrob Agents. 2006;28:366-9.

37. Poulakou G, Kontopidou FV, Paramythiotou E, Kompoti M, Katsiari M, Mainas E, et al. Tigecycline in the treatment of infections from multi-drug resistant gram-negative pathogens. J Infect. 2009;58:273-84.

38. Sheng WH, Wang JT, Li SY, Lin YC, Cheng A, Chen YC, et al. Comparative in vitro antimicrobial susceptibilities and synergistic activities of antimicrobial combinations against carbapenemresistant Acinetobacter species Acinetobacter baumannii versus Acinetobactergenospecies 3 and 13TU. Diagn Microbiol Infect Dis. 2011;70:380-6.

39. Daikos GL, Skiada A, Pavleas J, Vafiadi C, Salatas K, Tofas P, et al. Serum bactericidal activity of three different dosing regimens of colistin with implications for optimum clinical use. J Chemother. 2010;22(3):175-8.

40. Kontopidou F, Giamarellou H, Katerelos P, Maragos A, Kioumis I, Trikka-Graphakos E, et al. Infections caused by carbapenemresistant Klebsiella pneumoniae among patients in intensive care units in Greece: a multi-centre study on clinical outcome and therapeutic options. Clin Microbiol Infect. 2014;20(2):O117-23.

41. Durante-Mangoni E, Signoriello G, Andini R, Mattei A, De Cristoforo M, Murino P, et al. Colistin and rifampicin compared with colistin alone for the treatment of serious infections due to extensively drug-resistant Acinetobacter baumannii: a multicenter, randomized clinical trial. Clin Infect Dis. 2013;57(3):349-58.

42. Gaibani P, Lombardo D, Lewis RE, Mercuri M, Bonora S, Landini MP, Ambretti S. In vitro activity and post-antibiotic effects of colistin in combination with other antimicrobials against colistinresistant KPC-producing Klebsiella pneumoniae bloodstream isolates. J Antimicrob Chemother. 2014.

43. Akova M, Daikos GL, Tzouvelekis L, Carmeli Y. Interventional strategies and current clinical experience with carbapenemaseproducing Gram-negative bacteria. Clin Microbiol Infect. 2012;18(5):439-48.

44. Corvec S, Furustrand Tafin U, Betrisey B, Borens O, Trampuz A. Activities of fosfomycin, tigecycline, colistin, and gentamicin against extended-spectrum- $\beta$-lactamase-producing Escherichia coli in a foreign-body infection model. Antimicrob Agents Chemother. 2013;57(3):1421-7.

45. Bodmann KF, Heizmann WR, von Eiff C, Petrik C, Löschmann PA, Eckmann C. Therapy of 1,025 severely ill patients with complicated infections in a German multicenter study: safety profile and efficacy of tigecycline in different treatment modalities. Chemotherapy. 2012;58(4):282-94.

46. Michalopoulos AS, Livaditis IG, Gougoutas V. The revival of fosfomycin. Int J Infect Dis. 2011;15(11):e732-9.

47. Falagas ME, Giannopoulou KP, Kokolakis GN, Rafailidis PI. Fosfomycin: use beyond urinary tract and gastrointestinal infections. Clin Infect Dis. 2008;46(7):1069-77.

48. Levy Hara G, Gould I, Endimiani A, Pardo PR, Daikos G, Hsueh $\mathrm{PR}$, et al. Detection, treatment, and prevention of carbapenemaseproducing Enterobacteriaceae: recommendations from an International Working Group. J Chemother. 2013;25(3):129-40.

49. Livermore DM, Warner M, Mushtaq S, Doumith M, Zhang J, Woodford N. What remains against carbapenem-resistant Enterobacteriaceae? Evaluation of chloramphenicol, ciprofloxacin, colistin, fosfomycin, minocycline, nitrofurantoin, temocillin and tigecycline. Int J Antimicrob Agents. 2011;37(5):415-9.

50. Merino M, Poza M, Roca I, Barba MJ, Sousa MD, Vila J, Bou G. Nosocomial outbreak of a multiresistant Acinetobacter baumannii expressing OXA-23 carbapenemase in Spain. Microb Drug Resist. 2013.

51. Cuzon G, Bonnin RA, Nordmann P. First identification of novel NDM carbapenemase, NDM-7, in Escherichia coli in France. PLoS One. 2013;8(4):e61322. 
52. Tascini C, Sbrana F, Flammini S, Tagliaferri E, Arena F, Leonildi A, et al. Oral gentamicin gut decontamination for prevention of KPCproducing Klebsiella pneumoniae infections: relevance of concomitant systemic antibiotic therapy. Antimicrob Agents Chemother. 2014;58(4):1972-6.

53. Silvestri L, de la Cal MA, van Saene HK. Selective decontamination of the digestive tract: the mechanism of action is control of gut overgrowth. Intensive Care Med. 2012;38(11): 1738-50.

54. Oudemans-van Straaten HM, Endeman H, Bosman RJ, Attema-de Jonge ME, van Ogtrop ML, Zandstra DF, et al. Presence of tobramycin in blood and urine during selective decontamination of the digestive tract in critically ill patients, a prospective cohort study. Crit Care. 2011;15(5):R240.

55. Riddle V, Mason J, Cebrik D, Kostrub C, Fong L, Hillan K J. The effect of plazomicin injection on the QT/QTc interval in healthy volunteers. ICAAC 2013, poster \# A-017f. 1.

56. Walkty A, Adam H, Baxter M, Denisuik A, Lagacé-Wiens P, Karlowsky JA, Hoban DJ, Zhanel GG. In vitro activity of plazomicin against 5015 gram-negative and gram-positive clinical isolates obtained from patients in Canadian hospitals as part of the CANWARD study, 2011-2012. Antimicrob Agents Chemother. 2014.

57. Galani I, Souli M, Daikos GL, Chrysouli Z, Poulakou G, Psichogiou M, et al. Activity of plazomicin (ACHN-490) against MDR clinical isolates of Klebsiella pneumoniae, Escherichia coli, and Enterobacter spp. from Athens, Greece. J Chemother. 2012;24(4):191-4.

58. Tari LW, Li X, Trzoss M, Bensen DC, Chen Z, et al. Tricyclic GyrB/ParE (TriBE) inhibitors: a new class of broad-spectrum dualtargeting antibacterial agents. PLoS One. 2013;8(12):e84409.

59. Richter SG, Elli D, Kim HK, Hendrickx AP, Sorg JA, Schneewind $\mathrm{O}$, et al. Small molecule inhibitor of lipoteichoic acid synthesis is an antibiotic for Gram-positive bacteria. Proc Natl Acad Sci U S A. 2013;110(9):3531-6.

60. Bugli F, Cacaci M, Martini C, Torelli R, Posteraro B, Sanguinetti $\mathrm{M}$, et al. Human monoclonal antibody-based therapy in the treatment of invasive candidiasis. Clin Dev Immunol. 2013;2013: 403121.

61. Rizzello L, Pompa PP. Nanosilver-based antibacterial drugs and devices: mechanisms, methodological drawbacks, and guidelines. Chem Soc Rev. 2013. This paper propose some general guidelines for the design of antibacterial nanosystems and devices based on silver/nanosilver.

62.• Kruse EB, Aurbach U, Wisplinghoff H. Carbapenem-resistant Enterobacteriaceae: laboratory detection and infection control practices. Curr Infect Dis Rep. 2013. This review summarize the currently available laboratory methods and infection control options.

63. Ontario Agency for Health Protection and Promotion, Provincial Infectious Diseases Advisory Committee. Annex A-screening, testing and surveillance for antibiotic-resistant organisms (AROs). Annexed to: Toronto, ON: Queen's Printer for Ontario; 2013. 